\title{
Model-Based Insulin Sensitivity as a New Biomarker of Sepsis Diagnosis in the Intensive Care Unit
}

\author{
Wan Muhd Shukeri WF ${ }^{\mathrm{a}, \mathrm{b}}$, Jamaludin UK ${ }^{\mathrm{c}}$, Md Ralib $\mathrm{A}^{\mathrm{a}}$, Mat-Nor MB $^{\mathrm{a} *}$ \\ ${ }^{a}$ Department of Anaesthesiology and Intensive Care, Kulliyyah of Medicine, International Islamic University Malaysia, \\ ${ }^{b}$ Department of Anaesthesiology and Intensive Care, School of Medical Sciences, Universiti Sains Malaysia, Kelantan, Malaysia \\ 'Department of Mechanical Engineering, Universiti Malaysia Pahang, Pahang, Malaysia
}

\section{ABSTRACT}

INTRODUCTION: Currently, there is a lack of real-time biomarker to diagnose sepsis. Insulin sensitivity (SI) may be determined in real-time using mathematical glucose-insulin models, but its effectiveness as a diagnostic test of sepsis remains unexplored. We aimed to explore the diagnostic value of model-based SI as a new biomarker of sepsis in a mixed cohort of diabetic and non-diabetic patients newly admitted to the intensive care unit (ICU).

MATERIALS AND METHODS: In this cross-sectional study, we analysed SI levels derived from the IntensiveControl-of-Insulin-Nutrition-Glucose model in septic $(n=45)$ and non-septic $(n=41)$ patients upon their ICU admission. The diagnostic value of model-based SI for sepsis was determined through analysis of the area under the curve (AUC) of the receiver operating characteristic curve. RESULTS: Baseline SI levels were significantly lower in patients with sepsis than those without sepsis $\left(0.560\right.$ (SD=0.676) vs. 1.097 (SD=1.473) $\times 10^{-4} \mathrm{~L} / \mathrm{mU} / \mathrm{min}$, $P=0.037)$. However, the AUC of 0.588 revealed that model-based SI was a poor diagnostic test of sepsis in the mixed cohort of diabetics and non-diabetics. In a separate analysis among the non-diabetics $(n=19)$, model-based SI predicted sepsis with clinically valid performance (AUC 0.911). CONCLUSION: Presence of sepsis significantly reduced SI in the critically ill patients but a low SI could predict sepsis only in the non-diabetic cohort.

KEYWORDS: insulin sensitivity; insulin resistance; sepsis; intensive care units

\section{INTRODUCTION}

Insulin sensitivity (SI) can be defined as the capacity of cells to process glucose in response to insulin. ${ }^{1}$ Often used interchangeably with insulin resistance, the latter is the reciprocal concept of SI, which is defined by the condition that insulin works less effectively at lowering blood glucose (BG). Measurement of SI is usually of interest in the clinical investigations of diabetes mellitus (DM) and hypertension because of its key role in these diseases. Interestingly, SI is also known to decrease in sepsis, 2,3 although the exact mechanism is not fully

\section{Corresponding Author:}

Assoc. Prof. Dr. Mohd Basri Mat-Nor, Consultant Intensivist and Associate Professor, Department of Anaesthesiology and Intensive Care, Kulliyyah of Medicine, International Islamic University Malaysia, 25200 Kuantan, Pahang, Malaysia.

Tel No: +609-5912500

E-mail: basri.matnor@gmail.com understand. It has been postulated that sepsis alters the immuno-neuroendocrine axis and lipid and carbohydrate metabolisms, causing reduction in SI. ${ }^{4}$ Thus, as SI and sepsis are linked, it could prove to be an ideal metabolic biomarker for the diagnosis of sepsis.

Literature data specifies that the most efficient and scientifically correct methods for the evaluation of SI are "clamp" techniques. 5 The euglycemic hyperinsulinemic clamp technique is the reference method, the "gold standard" for quantifying SI in vivo because it directly measures the effects of insulin on glucose use under steady state conditions. ${ }^{6}$ However, largely because of the facts that the standard method for assessing SI is complex and costly to perform, several studies have been conducted to determine simpler and more applicable assessments. Therefore, physiological glucose -insulin system mathematical models for the evaluation of SI began to be used. An example of one such model 
is the Intensive-Control-of-Insulin-Nutrition-Glucose (ICING) model. ${ }^{7}$

The ICING model relates the rate of glucose decay to the concentration of insulin availability in the interstitium to assess SI. In the model's equations, there is only one parameter that needs to be identified which is SI of each patient. All other parameters are population constants based on prior studies and clinical data, i.e., BG levels, insulin infusion, and glucose input rates. SI can be derived at the bedside using application software incorporating such model and thus can provide real-time information to the clinicians. Derivation of SI does not involve extra procedures or costs outside of those required for BG control in the intensive care unit (ICU). Despite its simplicity and validity, the usefulness of SI derived from the ICING model as a diagnostic test of sepsis remains unexplored. The purpose of the present study was to explore the diagnostic value of model-based SI as a new biomarker of sepsis in a mixed cohort of diabetic and non-diabetic patients newly admitted to the ICU.

\section{MATERIALS AND METHODS}

\section{Study design and participants}

This cross-sectional study was conducted over a 1.5 year period, from 11th January 2017 to 31st July 2018, in the ICU of a university-affiliated hospital in Malaysia. This study was approved by the university research and ethics committee. All patients or their legally acceptable representatives gave written informed consents before participating in the study.

The inclusion criteria for this study were consecutive adult patients (aged 18 years or older) who received insulin infusion in the form of the Stochastic Targeted (STAR) glycaemic control protocol in the first 24 hour of their ICU admission, regardless of their DM status. The STAR protocol is a flexible, ICING model-based glycaemic control approach that directly accounts for intra- and inter-patient variability with a stochastically derived maximum 5\% risk of BG below $4 \mathrm{mmol} / \mathrm{L}$. The protocol recommends to perform BG level on admission and start the insulin infusion when BG exceeds $10 \mathrm{mmol} / \mathrm{L}$ for two consecutive readings, one hour apart. The aim was to maintain BG between 6 and IMJM Volume 20 No.2, Apr 2021
$10 \mathrm{mmol} / \mathrm{L}$. The protocol is made available to the users in the form of application software (STAR Glycaemic Controller.) Patients who received glycaemic control other than by the STAR protocol i.e. conventional insulin sliding scale or fixed insulin scale were excluded. Each patient cannot be enrolled twice into the study.

\section{Data Collection}

Data that was collected included age, sex, comorbidities, primary diagnosis, severity of illness as represented by Acute Physiology and Chronic Health Evaluation (APACHE II) score, degree of organ dysfunction as represented by Sequential Organ Failure Assessment (SOFA) scores, treatments received in the first 24 hours, presence of septic shock and blood culture results, BG levels, insulin infusion and glucose intake rates that the patients received. All patients had their HbA1C tested on admission to detect undiagnosed DM.

\section{Insulin sensitivity assessment}

SI assessment was done once at the first hour (baseline) of the patient's ICU admission. The SI levels of the patients were generated by fitting the BG measurements, insulin infusion and glucose intake rates into the ICING model incorporated in the STAR Glycaemic Controller software. The ICING model is represented by seven equations which are listed in the appendix. ${ }^{7}$ In these equations, there is only one parameter that needs to be identified which is SI of each patient. All other parameters are populations constants based on prior studies and clinical data, i.e. BG levels, insulin infusion and glucose input rates.

\section{Patients' Selection}

During the 1.5-year study period, a total of 105 patients were screened for eligibility (Figure 1). Nine (8.5\%) of the 105 patients received glycaemic control other than by the STAR protocol and were thus excluded from the study. A further $10(9.5 \%)$ of the 105 patients were excluded as they were readmission cases. The remaining $86(82 \%)$ of the 105 patients were eligible to be included into the analysis. Of these 86 patients, $41(47.7 \%)$ were classified as sepsis on admission and 45 (52.3\%) were classified as non-sepsis on admission. 


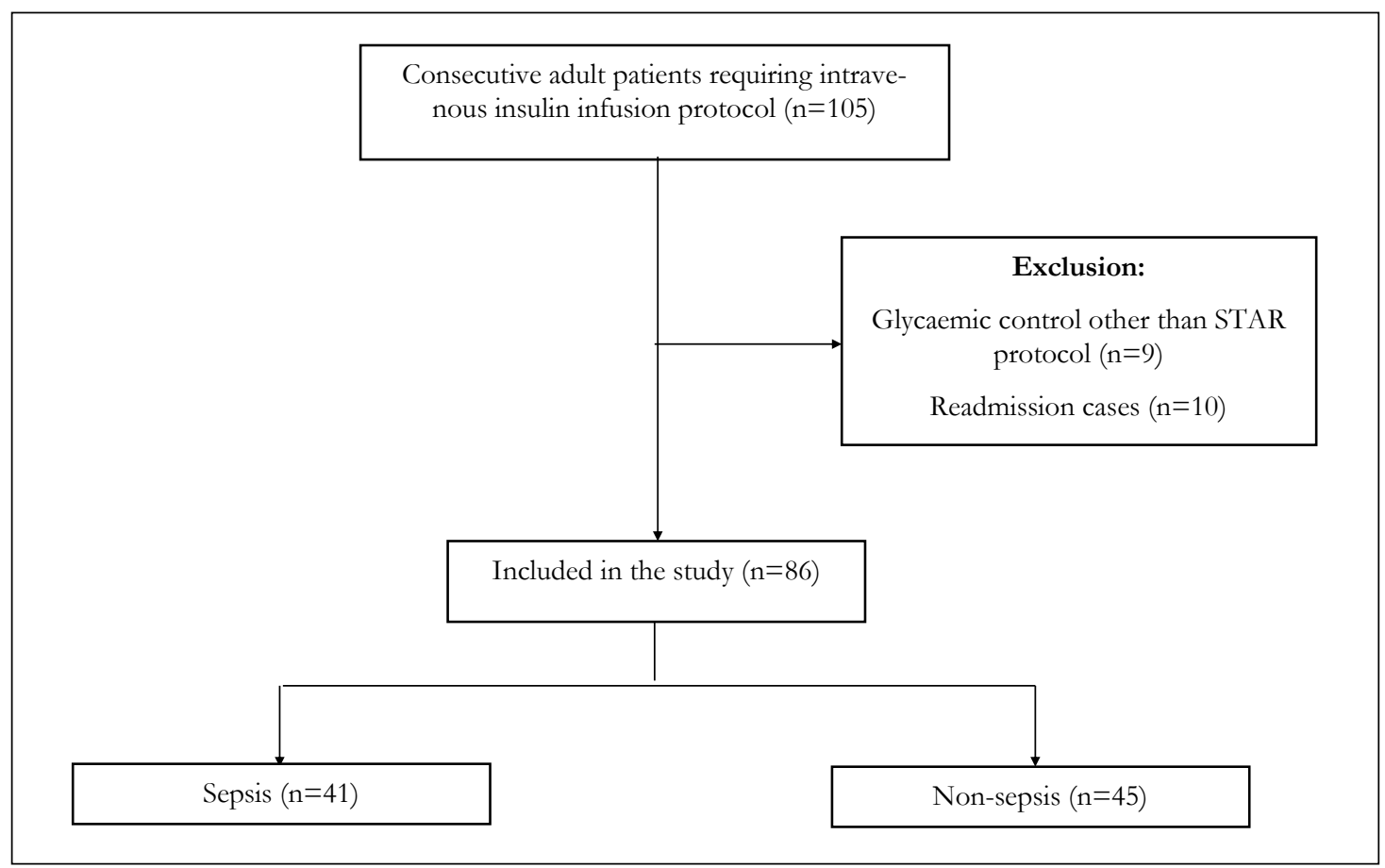

Figure 1. Flowchart of patients' selection

\section{Definitions}

Patients who were admitted with sepsis were confirmed clinically by the judgment of two senior intensive care clinicians. Sepsis was defined according to the recent Sepsis-3 criteria. ${ }^{8}$ Patients were classified as non-sepsis on admission if there was no clear clinical evidence of bacterial infections and the physicians in charge did not suspect it. In addition, blood culture or other specimens were negative. Patients were said to be diabetics if the presence of DM was known from their past medical history, or if this was newly diagnosed based on serum HbA1C screening on ICU admission, where a cut-off of 6.5\% was used (Ministry of Health Malaysia, 2009). ${ }^{9}$ Patients were said to be non-diabetics if the history of $\mathrm{DM}$ was not previously diagnosed, and if the $\mathrm{HbA} 1 \mathrm{C}$ screening on ICU admission was less than $6.5 \%$.

\section{Statistical analysis}

Data are presented as mean (standard deviation (SD)) or median (interquartile range (IQR)), and comparison of variables between the sepsis and non-sepsis groups was analysed using independent t-test or Mann-Whitney test. Normality of data was tested with Shapiro-Wilk test. Categorical variables are presented as frequency (percentage) and were compared with Chi-squared test.
The diagnostic performance of the model-based SI was assessed by the area under the curve (AUC) of the receiver operating characteristic curve (ROC) of the sensitivity against 1-specificity across a series of SI readings. The AUC ranges from 0.5 (no discrimination) to 1 (perfect discrimination). Clinical validity is assumed at an AUC of more than 0.7.10 The optimal cut-off point was defined as the measured quantity, which maximised sensitivity and specificity. The sensitivity, specificity and likelihood ratios (LR) of the test at the optimal cut-off point were calculated; these were reported with 95\% confidence interval (CI). Statistical analysis was performed using SPSS version 24.0 (IBM, Armonk, NY, USA) and MedCalc for Windows, version 17.5.5 (MedCalc Software, Ostend, Belgium).

\section{Sample size calculation}

In our previous study, SI within each subject group had a SD of $1.5 \times 10^{-4} \mathrm{~L} / \mathrm{mU} / \mathrm{min}^{11}$ If the true difference in the sepsis and the non-sepsis means is $2.4 \times 10^{-4} \mathrm{~L} /$ $\mathrm{mU} / \mathrm{min}$, we will need to study 41 sepsis subjects and 41 non-sepsis subjects to be able to reject the null hypothesis that the population means of the two groups are equal with probability (power) 0.8. The Type I error 
probability associated with this test of this null hypothesis is 0.05 .

\section{RESULTS}

\section{Clinical-demographic profiles}

The clinical demographic profiles of the included patients are presented in Table 1 . It was noted that the comorbid of chronic obstructive pulmonary disease was more prevalence in those with sepsis than in those without sepsis $(P=0.008)$. There was a significant association of respiratory disease $(P=0.016)$ and softtissue disease $(\mathrm{P}<0.0001)$ as the primary diagnoses of sepsis group as compared to the non-sepsis group. In contrast, there was a significant association of post-surgery $(P<0.0001)$ and cardiovascular disease $(P=0.016)$ as the primary diagnoses of non-sepsis group as compared to the sepsis group. Baseline APACHE II $(P=0.004)$ and SOFA score $(P=0.009)$ were significantly higher in those with sepsis than those without sepsis. A greater proportion of patients with sepsis received vasopressor support $(P$ 0.011) and renal replacement therapy $(P=0.011)$ in the first 24 hours than those without sepsis. In keeping with the primary diagnosis, more patients in the non-sepsis group had surgical intervention than those without sepsis $(P=0.005)$. In the sepsis group, the prevalence of septic shock was $71 \%$ and positive blood culture was noted in $17.8 \%$ of cases. Both groups had hyperglycaemia on admission but there was no significant difference in the level of BG between the sepsis and non-sepsis.

Table 1. Clinical-demographic profiles

\begin{tabular}{|c|c|c|c|}
\hline & $\begin{array}{c}\text { Non-sepsis } \\
(\mathrm{n}=45) \\
\mathrm{n}(\%)\end{array}$ & $\begin{array}{l}\text { Sepsis } \\
(\mathrm{n}=41) \\
\mathrm{n}(\%)\end{array}$ & $P$-value \\
\hline Demographic & & & \\
\hline $\begin{array}{l}\text { Age (years) a } \\
\text { Sex (male) }\end{array}$ & $\begin{array}{l}59(12) \\
19(46.3)\end{array}$ & $\begin{array}{l}60(12) \\
18(40)\end{array}$ & $\begin{array}{l}0.576^{b} \\
0.555^{c}\end{array}$ \\
\hline Clinical & & & \\
\hline $\begin{array}{l}\text { Comorbidities } \\
\text { Diabetes mellitus } \\
\text { Hypertension } \\
\text { Cardiovascular disease } \\
\text { Chronic kidney disease } \\
\text { COPD }\end{array}$ & $\begin{array}{l}33(80.5) \\
27(65.9) \\
3(7.3) \\
6(14.6) \\
0(0)\end{array}$ & $\begin{array}{l}34(75.6) \\
32(71.1) \\
10(22.2) \\
4(8.9) \\
7(15.6)\end{array}$ & $\begin{array}{l}0.582^{c} \\
0.600 \mathrm{c} \\
0.054^{c} \\
0.406^{c} \\
0.008^{c}\end{array}$ \\
\hline $\begin{array}{l}\text { Primary diagnosis } \\
\text { Respiratory } \\
\text { Soft tissue } \\
\text { Post-surgery } \\
\text { Gastrointestinal } \\
\text { Renal } \\
\text { Metabolic } \\
\text { CNS } \\
\text { Cardiovascular }\end{array}$ & $\begin{array}{l}6(14.6) \\
0(0) \\
20(48.8) \\
5(12.2) \\
0(0) \\
2(4.9) \\
3(7.3) \\
5(12.2)\end{array}$ & $\begin{array}{l}22(48.9) \\
12(26.7) \\
2(4.4) \\
3(6.7) \\
4(8.8) \\
3(6.7) \\
0(0) \\
0(0)\end{array}$ & $\begin{array}{l}0.001 \mathrm{c} \\
<0.0001^{\mathrm{c}} \\
<0.0001 \mathrm{c} \\
0.378^{\mathrm{c}} \\
0.051^{\mathrm{c}} \\
0.723^{\mathrm{c}} \\
0.065^{\mathrm{c}} \\
0.016^{\mathrm{c}}\end{array}$ \\
\hline $\begin{array}{l}\text { Severity of illness } \\
\text { APACHE II a } \\
\text { SOFA score }{ }^{a}\end{array}$ & $\begin{array}{l}14(7) \\
4(3)\end{array}$ & $\begin{array}{l}19(7) \\
6(3)\end{array}$ & $\begin{array}{l}0.004 \mathrm{~b} \\
0.009 \mathrm{~b}\end{array}$ \\
\hline $\begin{array}{l}\text { Treatment in first } 24 \text { hour } \\
\text { Vasopressor } \\
\text { Corticosteroids } \\
\text { Renal replacement therapy } \\
\text { Surgical intervention }\end{array}$ & $\begin{array}{l}18(43.9) \\
15(36.6) \\
7(17.1) \\
21(51.2)\end{array}$ & $\begin{array}{l}32(71.1) \\
21(46.7) \\
19(42.2) \\
10(22.2)\end{array}$ & $\begin{array}{l}0.011 \mathrm{c} \\
0.344^{\mathrm{c}} \\
0.011 \mathrm{c} \\
0.005^{\mathrm{c}}\end{array}$ \\
\hline Septic shock & - & $32(71)$ & - \\
\hline Positive blood culture & - & $8(17.8)$ & - \\
\hline Blood glucose $(\mathrm{mmol} / \mathrm{L})^{\mathrm{a}}$ & $13.9(3.3)$ & $14.9(5.5)$ & $0.290^{b}$ \\
\hline
\end{tabular}

CNS, central nervous system; COPD, chronic obstructive pulmonary disease; SAPS II, Simplified Acute Physiological Score II; SOFA, Sequential Organ Failure Assessment. a Mean (SD). ${ }^{\mathrm{b}}$ Independent $t$-test; c $\chi^{2}$-test. 


\section{Insulin sensitivity levels}

The means difference in the levels of SI between the sepsis and the non-sepsis group in the mixed cohort of diabetic and non-diabetic critically ill patients is shown in Figure 2. On admission to ICU, SI levels were significantly lower in patients who were diagnosed with sepsis than those without sepsis $(0.560(\mathrm{SD}=0.676)$ vs. $\left.1.097(\mathrm{SD}=1.473) \times 10^{-4} \mathrm{~L} / \mathrm{mU} / \mathrm{min}, \mathrm{P}=0.037\right)$.

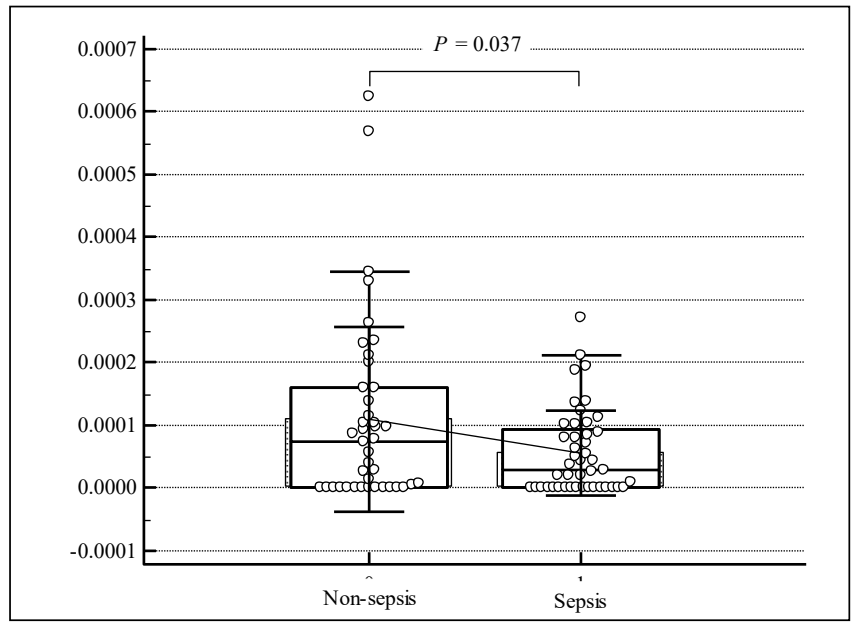

Note. The boxes show the means and error bars of $1 \mathrm{SD}$. The result of the comparison between the two groups was analysed by independent t-test

Figure 2. Baseline insulin sensitivity levels among sepsis and nonsepsis in a mixed cohort of diabetic and non-diabetic critically ill patients

\section{Diagnostic value of model-based insulin sensitivity}

Analysis of the ROC curve showed that the AUC of model-based SI was 0.588 (95\% CI: 0.477-0.693, $P=0.150$ ) (Figure 3). This result indicates that modelbased SI test had a poor diagnostic value for sepsis in the mixed cohort of diabetic and non-diabetic critically ill patients.

Subsequently, we attempted to analyse the diagnostic value of model-based SI according to the DM status of the patients, beginning with the non-diabetic patients. In the non-diabetic critically ill patients ( $n=19)$, SI was significantly lower in the sepsis compared to the non-sepsis group $(0.5166$ vs. $2.510 \times 10^{-4} \mathrm{~L} / \mathrm{mU} / \mathrm{min}, P=0.041$ ) (Figure 4 ). When the ROC curve was analysed, baseline SI predicted sepsis with a clinically valid performance (AUC 0.911 (95\% CI: $0.690-0.992), P<0.0001)$ in this group of patients. The ideal cut-off of the test

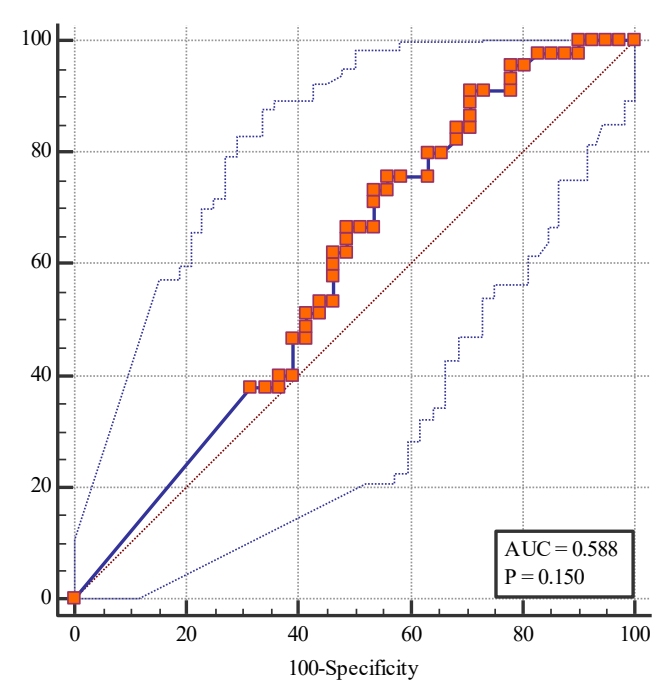

Figure 3. Receiver operating characteristic curve of baseline insulin sensitivity for discrimination of sepsis in a mixed cohort of diabetic and non-diabetic critically ill patients

was $0.880 \times 10^{-4} \mathrm{~L} / \mathrm{mU} / \mathrm{min}$. At this cut-off, the sensitivity was $90.00 \%$ (95\% CI: 55.5-99.7\%), specificity was $77.78 \%$ (95\% CI: $40.0-97.2 \%$ ), positive LR was 4.05 (95\% CI: 1.2-14.0) and negative LR was 0.13 (95\% CI: 0.02-0.9). After adjusting for SOFA score, baseline SI remained as an independent predictor of sepsis (adjusted OR 0.0001, $P=0.049$ ) in the non-diabetic cohort. This OR value means that patients with increasing SI had (1-0.001) or 99.9\% reduced chance to be associated with sepsis.

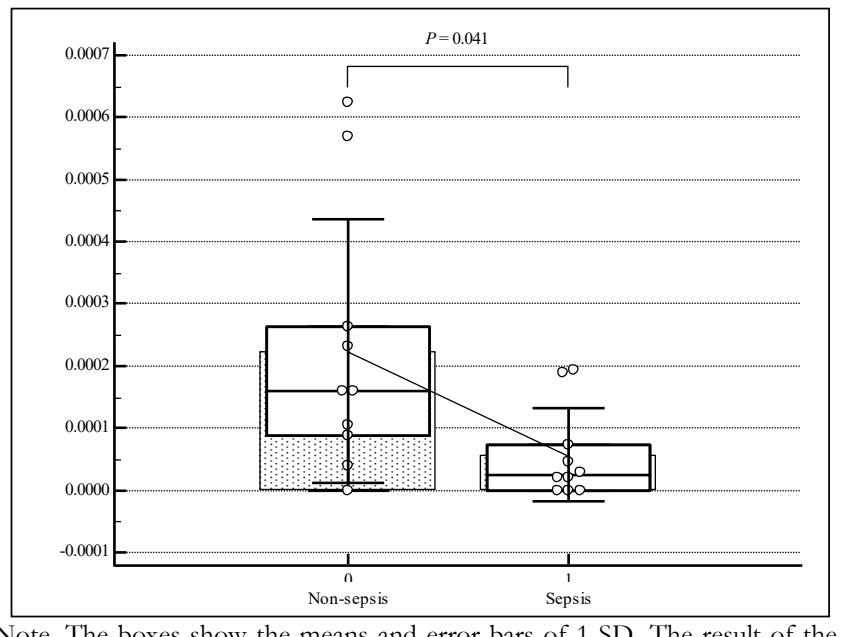

Note. The boxes show the means and error bars of 1 SD. The result of the comparison between the two groups was analysed by independent $t$-test.

Figure 4. Baseline SI levels among sepsis and non-sepsis in the non -diabetic critically ill patients

Meanwhile, in the diabetic critically ill patients ( $\mathrm{n}=67)$, there was no significant difference in the levels of SI between the sepsis and non-sepsis group $(0.574(\mathrm{SD}=$ $0.669)$ vs. $0.755(\mathrm{SD}=1.003) \times 10^{-4} \mathrm{~L} / \mathrm{mU} / \mathrm{min}, P=$ 0.390). In a ROC curve analysis, model-based SI 
predicted sepsis with a poor diagnostic performance (AUC 0.516 (95\% CI: $0.376-0.656), P=0.821$ ) in this group of patients.

\section{DISCUSSION}

\section{Clinical-Demographic Profiles}

Echoing the earlier studies conducted at our centre, most patients in the sepsis group had respiratory as their primary diagnostic class. ${ }^{12}$ This was followed by soft tissue as the second most common source of infection giving rise to sepsis. For the non-sepsis group, patients were admitted to ICU primarily due to post-surgical complications and a relatively higher proportion had cardiovascular as the primary diagnostic class than their sepsis counterparts. Patients with sepsis displayed greater severity of acute illness (APACHE II) and organ dysfunction (SOFA); this result is correlating with that observed in the earlier study conducted at our centre. ${ }^{12}$ In relation to the worse organ dysfunction with sepsis, it is expected that this group of patients required more organ support such as vasopressor administration and RRT as found in this study. Finally, both groups showed comparable degree of hyperglycaemia, similar to our earlier findings.

\section{Levels and Diagnostic Value of Model-Based Insulin Sensitivity}

Although SI was significantly lower in the presence of sepsis, evaluation of the ROC curve showed that modelbased SI failed to predict sepsis (AUC 0.588) when the diabetic and the non-diabetic critically ill patients were analysed together. This finding indicates that not only sepsis, but other severe illness and effects from the treatments received could be responsible for a low SI value in the general critically ill patients. While this result is disappointing, it is in line with the previous finding by Blakemore et al. (2008).13 The authors found that SI level is confounded by higher APACHEII score and thus concluded that a low SI is not a useful positive predictor of sepsis.

However, when the patients were analysed separately according to their DM status, low SI was found to be useful as a diagnostic test of sepsis in the non-diabetic critically ill patients, but not in those who were diabetic. If the utility of model-based SI is to be implemented in clinical setting, clinicians need to interpret the results in light of the patients' DM status. Our result so far indicates that SI test is of clinical applicability to the critically ill patients who were non-diabetic. Even in this group of patients, SI test is more useful to rule out rather than to diagnose sepsis, as suggested by the low negative LR of 0.13. Nonetheless, the low negative LR offers the clinical opportunity to avoid pre-emptive prescription of antibiotic or other treatment for sepsis and, as such, model-based SI is still a reasonably relevant diagnostic biomarker of sepsis in the non-diabetic critically ill patients. However, we obtained a lower cutoff value of $0.880 \times 10^{-4} \mathrm{~L} / \mathrm{mU} / \mathrm{min}$ in this study, compared to $2.869 \times 10^{-4} \mathrm{~L} / \mathrm{mU} / \mathrm{min}$ in the previous study ${ }^{11}$. This difference may be explained by the different criteria used to diagnose sepsis in the two studies.

\section{Strengths and limitations}

To the best of our knowledge, this is the first study to report the utility of model-based SI for sepsis diagnosis in a more representative ICU population i.e. one that includes both the diabetic and non-diabetic patients. Although the results are disappointing for those who were diabetic, SI test may still be reasonably useful for screening of sepsis in the non-diabetic critically ill cohort. The main limitation of this study is the sample size; whereby a relatively small number of 19 nondiabetic patients were included. This sample size did not permit a reliable multivariate analysis to be done. Also, as model-based SI was only studied in hyperglycaemic patients who received intravenous insulin infusion, our results is of limited clinical applicability to the general ICU population.

\section{CONCLUSION}

In this study, a significantly lower baseline SI value was noted in the presence of sepsis in a mixed cohort of diabetic and non-diabetic critically ill patients. However, model-based SI was found to be a poor predictor of sepsis when applied to this general cohort. Low SI can equally mark the presence of sepsis, other severe conditions or the result of treatment effects which are 
indicated in sepsis. Only when applied to the nondiabetic cohort that model-based SI may be useful as a negative predictor of sepsis, and less so as a positive predictor of sepsis.

\section{ACKNOWLEDGEMENTS}

The authors gratefully acknowledge the financial support provided by Ministry of Higher Education under Fundamental Research Grant SchemeFRGS/1/2018/TK03/UMP/02/3.

\section{CONFLICT OF INTEREST}

The authors declare we have no conflict of interest.

\section{APPENDIX}

$\dot{G}=-{ }_{p G} G(t)-S_{1} G(t) \frac{Q(t)}{1+\alpha_{G} Q(t)}+\frac{P(t)+E G P_{b}-C N S}{V_{G}}$

$\dot{Q}=n_{i}[I(t)-Q(t)]-n_{c} \frac{Q(t)}{1+\alpha_{G} Q(t)}$

$\dot{I}=-n_{K} I(t)-\frac{n_{L} I(t)}{1+\alpha_{t} I(t)}-n_{i}[I(t)-Q(t)]+\frac{u_{e x}(t)}{V_{I}}+\left(1-x_{L}\right) \frac{u_{e n}}{v_{t}}$

$\dot{P} 1=-d_{1} P 1+D(t)$

$\dot{P} 2=-\min \left(d_{2} P 2, P_{\max }\right)+d_{1} P 1$

$P(t)=\min \left(d_{2} P 2, P_{\max }\right)+P N(t)$

$u_{e n}(t)=k_{1} e^{-\frac{t(t)^{k_{2}}}{k_{3}}} ;$ when C-peptide data is not available

Where the nomenclatures are described below:

\begin{tabular}{|c|c|c|c|}
\hline Parameters & \multicolumn{2}{|l|}{ Description } & Unit \\
\hline$G$ & \multicolumn{2}{|l|}{ Blood glucose level } & $\mathrm{mmol} / \mathrm{L}$ \\
\hline$Q$ & \multicolumn{2}{|l|}{ Interstitial insulin level } & $\mathrm{mU} / \mathrm{L}$ \\
\hline$I$ & \multicolumn{2}{|l|}{ Plasma insulin level } & $\mathrm{mU} / \mathrm{L}$ \\
\hline$P 1$ & \multicolumn{2}{|l|}{ Stomach glucose content } & Mmol \\
\hline$P 2$ & \multicolumn{2}{|l|}{ Gut glucose content } & Mmol \\
\hline$u_{e n}$ & \multicolumn{2}{|l|}{ Endogenous insulin secretion rate } & $\mathrm{mU} / \mathrm{min}$ \\
\hline \multicolumn{4}{|c|}{ Parameters and kinetic values of ICING model } \\
\hline$E G P$ & Endogenous glucose production rate & 1.16 & mmol.min ${ }^{-1}$ \\
\hline CNS & Central nervous system glucose uptake & 0.3 & mmol.min ${ }^{-1}$ \\
\hline$P G$ & Patient endogenous glucose removal & 0.006 & $\min ^{-1}$ \\
\hline$S_{I}$ & \multicolumn{2}{|l|}{ Insulin sensitivity } & L.mu ${ }^{-1} \cdot \min$ \\
\hline$\alpha_{G}$ & Saturation parameter of insulin-mediated glucose & 0.0154 & L.mu ${ }^{-1}$ \\
\hline$V_{G}$ & Plasma glucose distribution volume & 13.3 & $\mathrm{~L}$ \\
\hline$n_{I}$ & Plasma-interstitium insulin diffusion rate & 0.006 & $\min ^{-1}$ \\
\hline$N_{C}$ & Receptor-bound insulin degradation & 0.006 & $\min ^{-1}$ \\
\hline$N_{K}$ & Renal insulin clearance & 0.0542 & $\min ^{-1}$ \\
\hline$n_{L}$ & Hepatic insulin clearance & 0.1578 & $\min ^{-1}$ \\
\hline$A_{I}$ & Saturation parameter for hepatic insulin clearance & 0.0017 & L.mu-1 \\
\hline$V_{I}$ & Insulin distribution volume & 4.0 & $\mathrm{~L}$ \\
\hline$X_{L}$ & First pass hepatic clearance & 0.67 & \\
\hline$D_{1}$ & \multirow{2}{*}{$\begin{array}{l}\text { Rate of glucose transport through the enteral route } \\
\text { into the blood stream }\end{array}$} & 0.0347 & $\min ^{-1}$ \\
\hline$D_{2}$ & & 0.0069 & $\min ^{-1}$ \\
\hline$P_{\max }$ & Maximal gut glucose flux & 6.11 & mmol.min ${ }^{-1}$ \\
\hline$U_{\max }$ & Maximal pancreatic secretion rate & 266.7 & mU.min \\
\hline$U_{\min }$ & Minimal pancreatic secretion rate & 16.7 & mU.min \\
\hline
\end{tabular}

Exogenous input variables of ICING model

$\begin{array}{lll}U_{e x} & \text { Intravenous insulin input rate } & \mathrm{mU} \cdot \mathrm{min}^{-1} \\ D & \text { Oral glucose input rate from enteral nutrition } & \mathrm{mmol} \cdot \mathrm{min}^{-1} \\ P N & \text { Intravenous glucose input rate from parenteral nutrition } & \mathrm{mmol} \cdot \mathrm{min}^{-1}\end{array}$




\section{REFERENCES}

1. Bergman RN, Ider YZ, Bowden CR, Cobelli C. Quantitative estimation of insulin sensitivity. AJP - Endocrinol Metab [nternet]. 1979;236(6):E667-77. Available from: http://ajpendo.physiology.org/cgi/content/ abstract/236/6/E667

2. Agwunobi a O, Reid C, Maycock P, Little R a, Carlson GL. Insulin resistance and substrate utilization in human endotoxemia. J Clin Endocrinol Metab. 2000;85 (10):3770-8.

3. Chambrier C, Laville M, Rhzioual Berrada K, Odeon M, Boulétreau P, Beylot M. Insulin sensitivity of glucose and fat metabolism in severe sepsis. Clin Sci (Lond) [Internet]. 2000;99(4):321-8. Available from: http:/ / www.ncbi.nlm.nih.gov/pubmed/10995598

4. Kajbaf F, Mojtahedzadeh M, Abdollahi M. Mechanisms underlying stress-induced hyperglycemia in critically ill patients. Therapy. 2007.

5. Mannucci E, Bardini G, Rotella F, Rotella CM. Comparison among different insulin sensitivity indices in obese patients. Diabet Med. 2003;20(6):462-6.

6. DeFronzo R a, Tobin JD, Andres R. Glucose clamp technique: a method for quantifying insulin secretion and resistance. Am J Physiol. 1979;237(3):E214-23.

7. Lin J, Razak NN, Pretty CG, Le Compte A, Docherty $\mathrm{P}$, Parente JD, et al. A physiological Intensive Control Insulin-Nutrition-Glucose (ICING) model validated in critically ill patients. Comput Methods Programs Biomed. 2011;102(2):192-205.

8. Singer M, Deutschman CS, Seymour CW, Shankar-Hari M, Annane D, Bauer M, et al. The Third International Consensus Definitions for Sepsis and Septic Shock (Sepsis-3). Jama [Internet]. 2016;315(8):801-10. Available from: http:/ /www.ncbi.nlm.nih.gov/ pubmed/26903338

9. Health M of. Clinical Practice Guidelines; Management of Type 2 Diabetes. Clin Pract Guidel Manag Type 2 Diabetes. 2009;73.

10. Bewick V, Cheek L, Ball J. Statistics review 13: receiver operating characteristic curves. Crit Care [Internet]. 2004;8(6):508-12. Available from: http:// www.ncbi.nlm.nih.gov/entrez/query.fcgi? cmd $=$ Retrieve $\& \mathrm{db}=$ PubMed\&dopt $=$ Citation\&list_uids $=15566624$

11. Shukeri WFWM, Mat-Nor MB, Jamaludin UK, Suhaimi F, Abd Razak NN, MD Ralib A. Levels and Diagnostic Value of Model-based Insulin Sensitivity in Sepsis: A Preliminary Study. Indian J Crit Care Med. 2018;22
(6):402-7.

12. Mat-Nor MB, MD Ralib A, Abdulah NZ, Pickering JW. The diagnostic ability of procalcitonin and interleukin-6 to differentiate infectious from noninfectious systemic inflammatory response syndrome and to predict mortality. J Crit Care. 2016;33:245-51.

13. Blakemore A, Wang S, Le Compte A, M Shaw G, Wong X-W, Lin J, et al. Model-based insulin sensitivity as a sepsis diagnostic in critical care. J Diabetes [Internet]. 2008;2(3):468-77. Available from: http:// www.ncbi.nlm.nih.gov/pmc/articles/PMC2769723/\% 5Cnhttp://www.pubmedcentral.nih.gov/ articlerender.foi? artid $=2769723 \&$ tool $=$ pmcentrez\&rendertype $=$ abstract 